\title{
Calculation of velocity structure functions for vortex models of isotropic turbulence
}

\author{
P. G. Saffman \\ Applied Mathematics 217-50, California Institute of Technology, Pasadena, California 91125 \\ D. I. Pullin \\ Graduate Aeronautical Laboratories 105-50, California Institute of Technology, \\ Pasadena, California 91125
}

(Received 1 March 1996; accepted 16 July 1996)

\begin{abstract}
Velocity structure functions $\overline{\overline{\left(u_{p}^{\prime}-u_{p}\right)^{m}}}$ are calculated for vortex models of isotropic turbulence. An integral operator is introduced which defines an isotropic two-point field from a volume-orientation average for a specific solution of the Navier-Stokes equations. Applying this to positive integer powers of the longitudinal velocity difference then gives explicit formulas for $\overline{\overline{\left(u_{p}^{\prime}-u_{p}\right)^{m}}}$ as a function of order $m$ and of the scalar separation $r$. Special forms of the operator are then obtained for rectilinear stretched vortex models of the Townsend-Lundgren type. Numerical results are given for the Burgers vortex and also for a realization of the Lundgren-strained spiral vortex, and comparison with experimental measurement is made. In an Appendix, we calculate values of the velocity-derivative moments for the Townsend-Burgers model. (C) 1996 American Institute of Physics. [S1070-6631(96)00611-3]
\end{abstract}

\section{STRUCTURE FUNCTIONS}

Pullin and Saffman, ${ }^{1}$ henceforth called (PS), and Pullin, Buntine, and Saffman, ${ }^{2}$ henceforth called (PBS), have studied the Lundgren-Townsend model of the fine scales of homogeneous turbulence. This model assumes that the statistics of the fine scales are approximated by the statistics of a random superposition of noninteracting vortex structures, each of which is a local, approximate or exact solution of the Navier-Stokes equations. Townsend, ${ }^{3}$ henceforth $T$, used steady axisymmetric Burgers vortices and Lundgren ${ }^{4}$ (henceforth $L$ ) employed unsteady, stretched spiral vortices. The remarkable feature of Lundgren's model is that with reasonable approximations, it leads naturally to a Kolmogorov $k^{-5 / 3}$ law for the velocity (energy) spectrum in an inertial subrange. PS and PBS examined the stretched-spiral model in more detail. They found higher order corrections to the energy spectrum and also calculated hyper flatness and skewness factors, defined as

$$
F_{2 p}=\frac{\overline{\overline{(\partial u / \partial x)^{2 p}}}}{\overline{\overline{\left((\partial u / \partial x)^{2}\right)^{p}}}}, \quad S_{2 p+1}=\frac{\overline{\overline{(\partial u / \partial x)^{2 p+1}}}}{\overline{\overline{\left((\partial u / \partial x)^{2}\right)^{(p+1) / 2}}}} .
$$

The double overline denotes ensemble averages, as in PS. Recall that for isotropic turbulence, $\overline{\overline{(\partial u / \partial x)^{2}}}=\epsilon / 15 \nu$, where $\epsilon$ is the ensemble average energy dissipation rate, $\nu$ is the kinematic viscosity, and $u$ is the component of velocity in the $x$-direction. Vorticity moments and the spectra of dissipation $E_{\epsilon \epsilon}$ and enstrophy $E_{\omega^{2} \omega^{2}}$ were also calculated.

Another set of physical quantities which has attracted considerable experimental and theoretical interest is the velocity structure functions. The longitudinal one of order $m$ is defined as

$$
B_{m}(r)=\overline{\overline{\left(u_{p}^{\prime}-u_{p}\right)^{m}}},
$$

where $u_{p}$ and $u_{p}^{\prime}$ are the velocity components at points $P$ and $P^{\prime}$ with coordinates $\mathbf{x}$ and $\mathbf{x}^{\prime}=\mathbf{x}+\mathbf{r}$, in the direction of $\mathbf{r}$, i.e.,

$$
u_{p}^{\prime}-u_{p}=\left(\mathbf{u}^{\prime}-\mathbf{u}\right) \cdot \overrightarrow{P P^{\prime}} / r .
$$

Note that unless the turbulence is forced to be statistically stationary in time, the structure functions will be time dependent, but we do not explicitly show the time dependence until a purpose is served by so doing.

One can also consider the transverse structure functions involving velocity components perpendicular to ${\overrightarrow{P P^{\prime}}}^{\prime}$, but we shall not calculate these in the present work.

\section{A. The isotropy integral}

Suppose we have a specific solution of the NavierStokes equations $\boldsymbol{u}(\boldsymbol{x}, t)$ in some structure-fixed Cartesian reference frame $\boldsymbol{x}$. We assume, without detailed discussion at present, that $\boldsymbol{u}(\boldsymbol{x}, t)$ satisfies conditions at infinity such that the integrals to be defined exist. Now let $\Phi\left(\mathbf{u}, \mathbf{u}^{\prime}, \mathbf{r}\right)$ be some scalar function of the velocities $\mathbf{u}=\mathbf{E} \cdot \boldsymbol{u}$ and $\mathbf{u}^{\prime}=\mathbf{E} \cdot \boldsymbol{u}^{\prime}$ at two points of separation $\mathbf{r}$. These velocities are measured in a fixed laboratory frame $\mathbf{x}$ and $\mathbf{E}(\Omega)$, the rotation matrix from the $\boldsymbol{x}$ to the $\mathbf{x}$ reference frame, is a function of the three Euler angles denoted symbolically by $\Omega$, which describe this rotation. Examples of $\Phi\left(\mathbf{u}, \mathbf{u}^{\prime}, \mathbf{r}\right)$ are integer powers of the longitudinal velocity difference defined in (3) or the difference between the dissipation at the two points. Isotropy, which is equivalent to uniform distribution of $\Omega$ on the unit sphere, will now be assumed. We define the isotropy integral as the volume-ensemble average of $\Phi$

$$
\overline{\overline{\Phi(r, t)}}=\frac{1}{L^{3}} \iint \Phi\left(\mathbf{u}(\mathbf{x}), \mathbf{u}^{\prime}(\mathbf{x}+\mathbf{r})\right) d \mathbf{x} d \Omega,
$$

where the six-dimensional integral is over the volume of a large box of size $L^{3}$ and over $\Omega$. We remark that we will generally follow the convention that Roman symbols denote representations of vectors or tensors in laboratory fixed axes, 
while italic symbols will refer to structure or vortex-fixed axes. Where scalar quantities or scalar functions for isotropic turbulence are considered, this distinction will not be required, but italic symbols will still be used. It should be clear that by construction $\overline{\overline{\Phi(r, t)}}$ represents an isotropic field. Further, we claim that, since $\boldsymbol{u}(\boldsymbol{x}, t)$ is a solution of the NavierStokes equations, fields obtained from (4) necessarily satisfy the statistical-dynamical equations for isotropic turbulence, for example, the Kármán-Howarth equation. It is possible to generalize (4) to three or more points, the results then being functions of several separations and of the geometrical parameters required to specify the relative orientation of the points. Examples would be three points forming a triangle or four points defining a nonplanar quadrilateral. In such cases the fields should satisfy all higher-order statistical-dynamical equations for isotropic turbulence.

\section{B. The second- and third-order structure functions}

There are two special cases of interest corresponding to $m=2$ and $m=3$. The second-order structure function, $m=2$, can be related to the shell-summed energy spectrum $E(k)$, where $k$ is wave number, by kinematic arguments which are independent of any model assumptions. The longitudinal correlation function $f(r)$ of isotropic turbulence, defined by

$$
\overline{\overline{u_{p}^{\prime} u_{p}}}=u^{2} f(r), \quad u^{2}=\frac{1}{\overline{3} \cdot \mathbf{u}},
$$

is related to the energy spectrum $E(k)$ by

$$
\frac{1}{2} u^{2}\left(3 f+r f^{\prime}\right)=\int_{0}^{\infty} \frac{\sin k r}{k r} E(k) d k,
$$

Batchelor. ${ }^{5}$ We can integrate (6) with respect to $r$ after multiplying by $r^{2}$, giving

$$
\frac{1}{2} u^{2} r^{3} f(r)=\int_{0}^{\infty}\left(\frac{\sin k r}{k^{3}}-\frac{r \cos k r}{k^{2}}\right) E(k) d k .
$$

Note that since $f(0)=1$, it follows from (6) that

$$
\int_{0}^{\infty} E(k) d k=\frac{3}{2} u^{2}
$$

The second-order longitudinal structure function is related to the correlation function by

$$
\overline{\overline{\left(u_{p}^{\prime}-u_{p}\right)^{2}}}=2 u^{2}(1-f(r)),
$$

and hence

$$
\begin{aligned}
B_{2}(r) \equiv \overline{\overline{\left(u_{p}^{\prime}-u_{p}\right)^{2}}}= & \frac{4}{r^{3}} \int_{0}^{\infty}\left(\frac{r^{3}}{3}+\frac{r \cos k r}{k^{2}}\right. \\
& \left.-\frac{\sin k r}{k^{3}}\right) E(k) d k .
\end{aligned}
$$

Kármán and Howarth ${ }^{6}$ showed that for isotropic turbulence, a relation between the second- and third-order longitudinal structure functions can be deduced from the Navier-Stokes equations. The equation can be written (Landau and Lifshitz, ${ }^{7}$ Sec. 33)

$$
\nu \frac{\partial B_{2}}{\partial r}-\frac{1}{6} B_{3}=\frac{2 \epsilon r}{15}+\frac{1}{2 r^{4}} \int_{0}^{r} r^{\prime 4} \frac{\partial B_{2}}{\partial t} d r^{\prime} .
$$

Alternative notations in terms of the longitudinal correlation functions are

$$
B_{2}(r)=2 u^{2}(1-f(r)), \quad B_{3}(r)=6 u^{3} k(r),
$$

where $k(r)$ is the triple velocity longitudinal correlation; $u^{3} k(r)=\overline{\overline{u_{p}^{\prime} u_{p}^{2}}}$.

Differentiating (11) after multiplying by $r^{4}$ gives the alternative form

$$
\begin{aligned}
\frac{\partial B_{2}}{\partial t} & -2 \nu\left(\frac{\partial^{2}}{\partial r^{2}}+\frac{4}{r} \frac{\partial}{\partial r}\right) B_{2}(r)+\frac{4 \epsilon}{3} \\
& =-\frac{1}{3 r^{4}} \frac{\partial}{\partial r}\left(r^{4} B_{3}(r)\right) .
\end{aligned}
$$

Neglecting unsteadiness and viscosity for large $r$, the last term on the left hand side of (11) balances the right hand side to give the asymptotic form for large $r$,

$$
B_{3}(r) \sim-\frac{4}{5} \epsilon r
$$

$\left(\operatorname{Kolmogorov}^{8}\right)$. This, of course, also follows from (13).

In what follows, we obtain in Sec. II the form of the integral operator (4) for stretched-vortex models, followed in Sec. III by evaluation for the $T$ model. Section IV considers the $L$ stretched-spiral vortex model. In Appendix A we calculate the longitudinal velocity-derivative moments for the $T$ model. A $k$-space approach to the calculation of the thirdorder structure function is outlined in Appendix B.

\section{STRETCHED-VORTEX MODELS}

We now specialize to stretched-vortex solutions of the Navier-Stokes equations. The vorticity is supposed to be aligned in straight uniform tubes. In a cylindrical polar coordinate system $(s, \theta, z)$ fixed in a tube, with $z$-axis along the tube, the vorticity induces a tangential velocity field $u_{\theta}(s, \theta, t)$ at $P$, where the Cartesian coordinates of $P$ in the structure fixed coordinate system are

$$
x=s \cos \theta, \quad y=s \sin \theta, \quad z,
$$

and those of $P^{\prime}$ are

$$
\begin{aligned}
& x^{\prime}=s^{\prime} \cos \theta^{\prime}=x+r \sin \alpha \cos \beta, \\
& y^{\prime}=s^{\prime} \sin \theta^{\prime}=y+r \sin \alpha \sin \beta, \\
& z^{\prime}=z+r \cos \alpha,
\end{aligned}
$$

where $0<\alpha<\pi, 0<\beta<2 \pi$ ( $\alpha$ and $\beta$ are co-latitude and longitude of $\overrightarrow{P P^{\prime}}$ ). The external straining field acting on the vortex is

$$
\boldsymbol{u}_{s}=\left(-\frac{1}{2} a x,-\frac{1}{2} a y, a z\right) .
$$

Then, assuming that the radial component of the vorticity induced velocity field is to a good approximation negligible, the vortex contribution $(v)$ to the velocity difference is found from (3) to be 
$\delta u_{v}=\left.\left(u_{p}^{\prime}-u_{p}\right)\right|_{v}=\left(u_{\theta} \sin \theta-u_{\theta}^{\prime} \sin \theta^{\prime}\right) \sin \alpha \cos \beta$

$$
-\left(u_{\theta} \cos \theta-u_{\theta}^{\prime} \cos \theta^{\prime}\right) \sin \alpha \sin \beta \text {. }
$$

Here, $u_{\theta}^{\prime}$ is evaluated at $s^{\prime}, \theta^{\prime}$. In addition there is a contribution from the background external straining field $(s)$, found from (3) using the external strain (17)

$$
\begin{aligned}
\delta u_{s}=\left.\left(u_{p}^{\prime}-u_{p}\right)\right|_{s}= & -\frac{1}{2} \operatorname{ar} \sin ^{2} \alpha \cos ^{2} \beta-\frac{1}{2} \operatorname{ar} \sin ^{2} \alpha \\
& \times \sin ^{2} \beta+\operatorname{ar} \cos ^{2} \alpha \\
= & \operatorname{ar}\left(\cos ^{2} \alpha-\frac{1}{2} \sin ^{2} \alpha\right) .
\end{aligned}
$$

The longitudinal velocity difference is

$$
\delta u=\delta u_{v}+\delta u_{s} .
$$

The $m$ th order structure function is the five-dimensional integral of $\delta u$, obtained by integrating over all orientations, all positions in space and the lifetime $\left(t_{1}, t_{2}\right)$ of the structure,

$$
\begin{gathered}
\int_{t_{1}}^{t_{2}} N e^{a t} d t \int_{0}^{\infty} s d s \int_{0}^{2 \pi} d \theta \int_{0}^{\pi} \frac{\sin \alpha d \alpha}{2} \\
\times \int_{0}^{2 \pi} \frac{d \beta}{2 \pi}\left(\delta u_{v}+\delta u_{s}\right)^{m}
\end{gathered}
$$

Equation (21) differs from (4) as follows; first, owing to the assumption of isotropy, integration over the third Euler angle which gives a rotation about the $z$ axis equivalent to a shift in the origin of $\theta$, can be done explicitly. Second, since by assumption the vorticity is independent of $z$, integration in the $z$ direction can be represented as multiplication by the vortex length at time $t, l_{0} e^{a t}$ where $l_{0}$ is the initial vortex length at the instant of its creation and $e^{a t}$ is the stretch. Third, the volume integral in (4) is replaced by a summation over all vortex structures which, it is supposed, form the turbulence in $L^{3}$ at time $t$. Use of an ergodic hypothesis then allows replacement of the sum with an integral over the lifetime of an individual structure weighted by the quantity $N_{c}$, the number of structures created per unit time in the volume $L^{3}$ - see Reference 4 for a detailed discussion. The parameters $L, l_{0}$ and $N_{c}$ are then grouped together as $N=N_{c} l_{0} / L^{3}$, the average rate of creation of vortex length per unit volume per unit time. This has dimensions $[\text { length }]^{-2}[\text { time }]^{-1}$.

We now encounter the problem, associated with infinite stretched vortex models, that the integral (21) diverges because $\delta u_{s}$ is unbounded. We handle this difficulty by assuming that we can add and subtract $\left(\delta u_{s}\right)^{m}$, giving

$$
\begin{aligned}
B_{m}(r)= & \int_{t_{1}}^{t_{2}} N e^{a t} d t \int_{0}^{\infty} s d s \int_{0}^{2 \pi} d \theta \int_{0}^{\pi} \frac{\sin \alpha d \alpha}{2} \\
& \times \int_{0}^{2 \pi} \frac{d \beta}{2 \pi}\left[\left(\delta u_{v}+\delta u_{s}\right)^{m}-\left(\delta u_{s}\right)^{m}\right]+\overline{\overline{\left(\delta u_{s}\right)^{m}}}
\end{aligned}
$$

The integrand is now bounded and in general convergent. The ensemble average of the last term in (22) is regarded as a homogeneous spatial average over all space and has the value from (19)

$$
\begin{aligned}
\overline{\overline{\left(\delta u_{s}\right)^{m}} \equiv b_{m}^{(s)}(r)=} & \frac{1}{2} a^{m} r^{m} \\
& \times \int_{0}^{\pi} \sin \alpha\left(\cos ^{2} \alpha-\frac{1}{2} \sin ^{2} \alpha\right)^{m} d \alpha \\
= & a^{m} r^{m} k_{m},
\end{aligned}
$$

where $k_{2}=1 / 5, k_{3}=2 / 35, k_{4}=3 / 35, k_{5}=4 / 77, k_{6}=53 / 1001$, $k_{7}=6 / 143, k_{8}=95 / 2431$ etc. The integral in (22) defines the function $b_{m}(r)$, so that

$$
B_{m}(r)=b_{m}(r)+b_{m}^{(s)}(r),
$$

where the first term on the right hand side contains contributions from the vorticity and strain in the structure and the last term is due to pure strain alone.

\section{THE TOWNSEND MODEL}

\section{A. The $m$ th-order structure functions}

To proceed further, we need an expression for the vorticity induced, tangential velocity $u_{\theta}$. We begin with calculations for the symmetric Burgers vortex, used in $T$ as a model of the fine scale structure. The vorticity and tangential velocity are respectively

$$
\begin{aligned}
& \omega(s)=\frac{\Gamma a}{4 \pi \nu} e^{-a s^{2} / 4 \nu}, \\
& u_{\theta}(s)=\frac{\Gamma}{2 \pi s}\left(1-e^{-a s^{2} / 4 \nu}\right),
\end{aligned}
$$

where $s$ is the dimensional distance from the axis of the vortex. Steady asymmetric Burgers vortices were calculated by Robinson and $\mathrm{Saffman}^{9}$ for moderate values of the Reynolds number $\Gamma / \nu$, and for large values by Moffatt, Kida and Ohkitani. ${ }^{10}$

We now put $\xi=s / r_{0}$, and define $\tilde{r}=r / r_{0}$ where $r_{0}$ $=\sqrt{\nu / a}$. For the Burgers vortex, for which the radial velocity is zero, and $u_{\theta}$ does not depend explicitly on $\theta$, we have, after some algebra

$$
\begin{aligned}
& \delta u_{v}=\sin \alpha \sin (\beta-\theta)\left(u_{\theta}^{\prime} \frac{\xi}{\xi^{\prime}}-u_{\theta}\right), \\
& \xi^{\prime}=\left[\xi^{2}+\widetilde{r}^{2} \sin ^{2} \alpha+2 \xi \tilde{r} \sin \alpha \cos (\beta-\theta)\right]^{1 / 2},
\end{aligned}
$$

where $u_{\theta}$ and $u_{\theta}^{\prime}$ are (26) evaluated at $s=r_{0} \xi$ and $s^{\prime}=r_{0} \xi^{\prime}$, respectively. In obtaining (27-28) we have used (18) and $\xi^{\prime}$ $\sin \left(\beta-\theta^{\prime}\right)=\xi \sin (\beta-\theta)$ which follows from (16). Note that $\tilde{r}=r / r_{0}$ is the dimensionless separation of the points at which the velocity is measured. Since $\delta u_{v}$ can be seen to be a function of $\xi, \alpha$ and $\beta-\theta$ only, performing the time integral in $(21)$ with $\left(t_{1}, t_{2}\right)=\left(0, t_{2}\right)$ then gives 


$$
\begin{aligned}
b_{m}(r)= & \frac{N \tau_{2} \nu}{4 \pi a} \int_{0}^{\infty} \int_{0}^{\pi} \int_{0}^{2 \pi}\left(\left(\delta u_{v}+\delta u_{s}\right)^{m}-\left(\delta u_{s}\right)^{m}\right) \\
& \times \xi d \xi \sin \alpha d \alpha d(\beta-\theta),
\end{aligned}
$$

where

$$
\tau_{2}=\frac{1}{a}\left(e^{a t_{2}}-1\right) .
$$

Since the $T$ vortices are steady, we assume they have the same structure (age), but the total length will again increase exponentially with time. Owing to this exponential growth, a cutoff, at time denoted by $t_{2}$ will be required to give statistically stationary results for the structure functions. This is discussed further just prior to (44). The pure strain contribution $\delta u_{s}$ is still given by (19).

\section{B. Second- and third-order structure functions for the Townsend model}

Using the "Lundgren filter" (PS, Sec. 11), which can be evaluated in closed form for this velocity field, gives an energy spectrum

$$
E(k)=\frac{N \tau_{2} \Gamma^{2}}{4 \pi k} e^{-2 k^{2} \nu / a} .
$$

The dissipation can be calculated directly from the formula,

$$
\epsilon=2 \nu e_{i j}^{2}=3 \nu a^{2}+\nu N \tau_{2} \int_{0}^{\infty} 2 \pi s \omega^{2}(s) d s,
$$

where $\omega(s)$ is given by (25). This gives

$$
\epsilon=3 \nu a^{2}+\frac{N \tau_{2} \Gamma^{2} a}{8 \pi},
$$

where the first term on the right-hand side comes from the background. Note also that the structure contribution is equal to $2 \nu \int_{0}^{\infty} k^{2} E(k) d k$, where $E(k)$ is given by (31).

This spectrum (31) has an infrared divergence, which prevents one from obtaining a relation between the model parameters, $N, \tau_{2}$ and $\Gamma$ (the circulation of each structure), and the mean square velocity. But (31) gives a convergent expression for the second-order structure function when substituted into (10),

$$
\begin{aligned}
\overline{\overline{\left(u_{p}^{\prime}-u_{p}\right)^{2}}}= & \frac{N \tau_{2} \Gamma^{2}}{\pi} \int_{0}^{\infty}\left(\frac{1}{3}+\frac{\cos z}{z^{2}}-\frac{\sin z}{z^{3}}\right) \\
& \times e^{-2 z^{2} / \zeta^{2}} \frac{d z}{z},
\end{aligned}
$$

where $\zeta=\sqrt{a r^{2} / \nu}$. Attempts to evaluate the integral (34) in closed form were unsuccessful, but numerical integration is straightforward. Note that

$$
\frac{1}{3}+\frac{\cos z}{z^{2}}-\frac{\sin z}{z^{3}}=\frac{z^{2}}{30}-\frac{z^{4}}{840}+\frac{z^{6}}{45360}+O\left(z^{8}\right) .
$$

As $r \rightarrow \infty$, the behavior of $\overline{\overline{\left(u_{p}^{\prime}-u_{p}\right)^{2}}}$ as given by (34), asymptotes to $\left(2 N \tau_{2} \Gamma^{2} / 3 \pi\right) \log r$, and there does not appear to be a significant range in which $\overline{\overline{\left(u_{p}^{\prime}-u_{p}\right)^{2}}}$ has a power law dependence on $r$. The dimension of $N \tau_{2}$ is $1 /$ (length) $)^{2}$. Using (35) in the integrand of (34) gives

$\overline{\overline{\left(u_{p}^{\prime}-u_{p}\right)^{2}}}=N \tau_{2} \Gamma^{2}\left(\frac{\tilde{r}^{2}}{120 \pi}-\frac{\tilde{r}^{4}}{6720 \pi}+\frac{\tilde{r}^{6}}{362880 \pi}+O\left(\tilde{r}^{8}\right)\right)$,

where we recall that $\widetilde{r}=r / r_{0}$ and $r_{0}=\sqrt{\nu / a}$.

We now use (29) for the $T$ model velocity field to calculate $b_{2}(r)$ and $b_{3}(r)$. For small $r$, we obtain for $b_{2}(r)$ an expression which agrees with (36). Again from (24) but with the exponent 2 replaced by 3 , we obtain

$$
b_{3}(r)=N \tau_{2} \Gamma^{2}(a \nu)^{1 / 2}\left(-\frac{\tilde{r}^{3}}{140 \pi}+\frac{\tilde{r}^{5}}{6720 \pi}+O\left(\tilde{r}^{7}\right)\right) .
$$

We now take account of the background strain, and take

$$
\begin{aligned}
& B_{2}(r)=b_{2}(r)+\frac{1}{5} a^{2} r^{2}, \\
& B_{3}(r)=b_{3}(r)+\frac{2}{35} a^{3} r^{3} .
\end{aligned}
$$

It should be possible to transform the three-dimensional integral in (29) for $m=2$ into the one-dimensional integral (34), but this has not been done to date. (Numerical investigation of the integrals (29), for $m=2$, and (34) has shown that they are the same to a high degree of accuracy, apart from the background contribution. Note that the contribution of the interaction terms $\delta u_{v} \delta u_{s}$ in (29) is not obviously zero, but does vanish because $\delta u_{v}$ can be shown to be odd in $(\theta-\beta)$.

We remark here that we expect (11) or (13), integrated in the interval $\left(t_{1}, t_{2}\right)$, to be satisfied by (22) with $m=2$, $m=3$, respectively. Alternatively (11) and (13) should themselves be satisfied by a form of (22) for which the erogodic hypothesis, which it may be recalled introduces the time integration and the factor $N_{c}$ contained in the parameter $N$, is not applied. Using the above results we have verified this for the Townsend-Burgers model to $O\left(r^{8}\right)$. Although the Burgers vortices are strictly steady-flow solutions of the NavierStokes equations, it was found to be necessary to retain the time dependence in (29) in order to satisfy the KármánHowarth equation. This is surprising.

\section{Numerical evaluation of the structure-function integrals}

For the purposes of numerical evaluation of (29) for the $T$ model, we nondimensionalize $B_{m}(\widetilde{r})$ against $(\epsilon \nu)^{m / 4}$ and velocities in the integrand by $\Gamma / r_{0}$. After some manipulation we obtain

$$
\begin{aligned}
\overline{\overline{\left(u_{p}^{\prime}-u_{p}\right)^{m}}} & =\frac{1}{(\epsilon \nu)^{m / 4}}\left(\frac{N \tau_{2} \nu}{a}\right)\left(\frac{\Gamma}{\nu}\right)^{m}\left(\frac{a^{2} \nu}{\epsilon}\right)^{m / 4} \\
& \times \int_{0}^{\infty} \xi d \xi \int_{0}^{2 \pi} d(\beta-\theta) \int_{0}^{\pi} \sin \alpha d \alpha
\end{aligned}
$$


TABLE I. Calculated values of the velocity derivative moments $F_{2 p}$ and $S_{2 p+1}$ for the Townsend-Burgers model, for three values of $\Gamma / \nu$.

\begin{tabular}{rcccccc}
\hline \hline$\frac{\Gamma}{\nu}$ & $S_{3}$ & $F_{4}$ & $S_{5}$ & $F_{6}$ & $S_{7}$ & $F_{8}$ \\
\hline 100 & -0.499 & 0.523 & -0.329 & 0.309 & -0.239 & 0.226 \\
500 & -0.117 & 0.216 & -0.051 & 0.080 & -0.027 & 0.038 \\
1000 & -0.060 & 0.205 & -0.025 & 0.074 & -0.014 & 0.033 \\
\hline \hline
\end{tabular}

$$
\begin{aligned}
& \times\left\{\left[\delta \hat{U}_{v}+\left(\frac{\Gamma}{\nu}\right)^{-1} \widetilde{r} G(\alpha)\right]^{m}\right. \\
& \left.-\left(\frac{\Gamma}{\nu}\right)^{-m} \tilde{r}^{m} G^{m}(\alpha)\right\}+\tilde{r}^{m} k_{m}\left(\frac{a^{2} \nu}{\epsilon}\right)^{m / 4},
\end{aligned}
$$

where

$$
\begin{aligned}
& \hat{U}_{v}(\xi)=\frac{1}{2 \pi \xi}\left(1-e^{-\xi^{2} / 4}\right), \quad G(\alpha)=1-\frac{3}{2} \sin ^{2} \alpha, \\
& \delta \hat{U}_{v}=\sin \alpha \sin (\beta-\theta)\left(\hat{U}_{v}\left(\xi^{\prime}\right) \frac{\xi}{\xi^{\prime}}-\hat{U}_{v}(\xi)\right),
\end{aligned}
$$

and $\xi^{\prime}$ is given by (28). The right-hand side of (40) is a function of four parameters; the three dimensionless groups $N \tau_{2} \nu / a, \Gamma / \nu, a^{2} \nu / \epsilon$ and the dimensionless separation $\widetilde{r}$. This can be reduced to three parameters by use of (33) for the dissipation, which when divided by $\nu a^{2}$ gives

$$
\left(\frac{a^{2} \nu}{\epsilon}\right)^{-1}=\frac{1}{8 \pi}\left(\frac{N \tau_{2} \nu}{a}\right)\left(\frac{\Gamma}{\nu}\right)^{2}+3
$$

The group $N \tau_{2} \nu / a$ may be viewed as a parameter related to the volume-filling fraction of the Burgers vortices. We envision a statistical equilibrium as outlined in $L$ (Sec. III B) whereby vortices are created, stretched by the strain provided by larger scales, and then destroyed by processes which we view as outside the domain of the present model. The quantity $N \tau_{2}$, representing a cutoff at some time $t_{2}$, may then be interpreted as an average total length of vortex per unit volume. This length may be made up of from the sum of the contribution of many short vortices of varying lengths, each experiencing instantaneous stretching. As this stretchng process takes place, some vortices are destroyed thus maintaining the equilibrium. If the cross sectional area of a vortex is $A$, the volume fraction occupied by the vortices is $A N \tau_{2}$. If this is chosen as its upper bound of unity, i.e. the vortices are closely packed and fill space, and if we estimate from (25) the radius of a vortex as $2 r_{0}=2(\nu / a)^{1 / 2}$, then $A=\pi\left(2 r_{0}\right)^{2}$ gives

$$
\frac{N \tau_{2} \nu}{a}=\frac{1}{4 \pi} .
$$

The integrals in (40) were calculated by dividing the infinite interval for $\xi$ into two intervals $0 \leqslant \xi \leqslant \xi_{\max }$ and $\xi_{\max }<\xi \leqslant \infty$. In the first of these the triple integral was evaluated numerically in the box $0 \leqslant \xi \leqslant \xi_{\max }, 0 \leqslant \beta \leqslant 2 \pi$, $0 \leqslant \alpha \leqslant \pi / 2$ using the $M$-point periodic trapezoidal rule in the $\beta$-direction and an $M$-point Simpson's rule in both the $\xi$ and the $\alpha$ directions. Note that the integrand can be shown to be symmetric about $\alpha=\pi / 2$. An asymptotic correction for the interval $\xi_{\max }<\xi \leqslant \infty$ was obtained by neglecting the exponential term in the first of (41) and expanding the resulting integrand in (40) (for each $\mathrm{m}$ ) in powers of the small quantity $\widetilde{r} / \xi, \xi>\xi_{\max }$. The resulting series of triple integrals can then each be done analytically. This procedure requires both $\xi_{\max } \gg 1$ and $\widetilde{r} / \xi_{\max } \ll 1$. Numerical results obtained using $M=256$ are believed accurate to four figures. We take $N \tau_{2} \nu / a$ given by (44), choose a value of $\Gamma / \nu$ and calculate $a^{2} \nu / \epsilon$ from (43). The numerical results were checked by several methods. First, almost all calculations reported in this paper were done independently by both authors. Second, for $\widetilde{r} \ll 1$, dividing by $\widetilde{r}^{n}$ gives estimates of the longitudinal velocity gradients. These were then compared with a calculation of the velocity gradients obtained by an independent method (see Appendix A and Table I) giving four-figure agreement. A third check is to compare the numerical $b_{2}(r)$ and $b_{3}(r)$ with (36) and (37) for $\widetilde{r}$ that is not too large. A fourth check is to compare numerical values of $b_{2}(r)$ with numerical evaluation of (34). This gave four-figure agreement over the range $0<\tilde{r}<20$.

Velocity structure functions from numerical evaluation of (40) were calculated over the range $0<\tilde{r} \leqslant 10$, for $\Gamma / \nu=10^{2}, 5 \times 10^{2}$ and $10^{3}$. The even structure functions for $m=2, m=4$ and $m=8$ are shown in Figure 1. The abscissa is $r / \eta$, which for given $\widetilde{r}$ can be calculated from

$$
\frac{r}{\eta}=\widetilde{r}\left(\frac{a^{2} \nu}{\epsilon}\right)^{-1 / 4} .
$$

Also shown are data of Tabeling ${ }^{11}$ at a Taylor Reynolds number $R_{\lambda}=507$. These data were obtained from an experiment in low temperature helium gas contained in a confined cylinder. ${ }^{12}$ The end disks of the cylinder were fitted with blades and the turbulence was produced by rotating these disks in opposite directions. The Townsend-Burgers model calculations are asymptotic to the data for $m=2$. This can be interpreted as a result of the use of (34) and of isotropy, which together guarantee that for small separations, the second-order structure function must satisfy

$$
\overline{\overline{\left(u_{p}^{\prime}-u_{p}\right)^{2}}} \frac{1}{(\epsilon \nu)^{1 / 2}}=\tilde{r}^{2} .
$$

This result is equivalent to the requirement that the righthand side of (A5a) must identically equal unity, and is therefore a consistency check of the model. The calculations do not otherwise show good agreement with the data, with the discrepancy increasing to three orders of magnitude for 
(a)

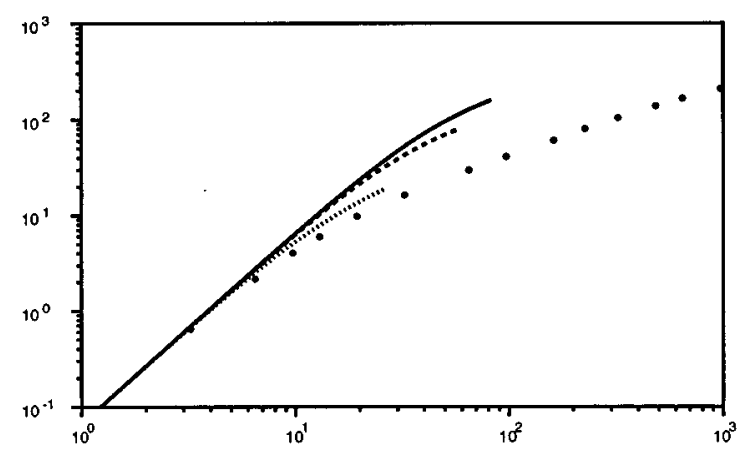

(b)

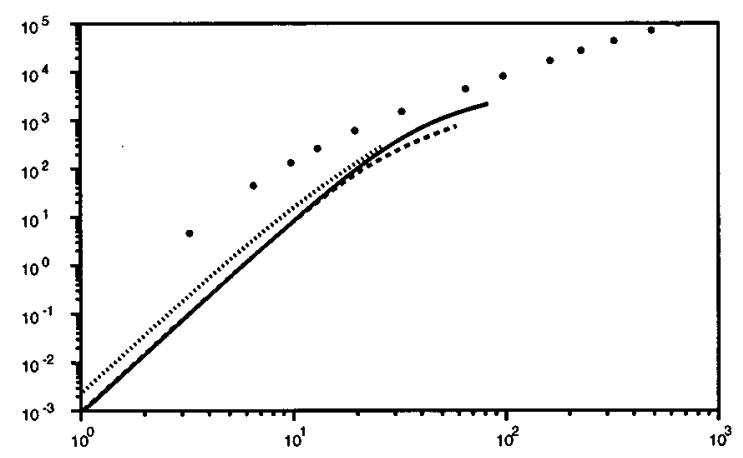

(c)

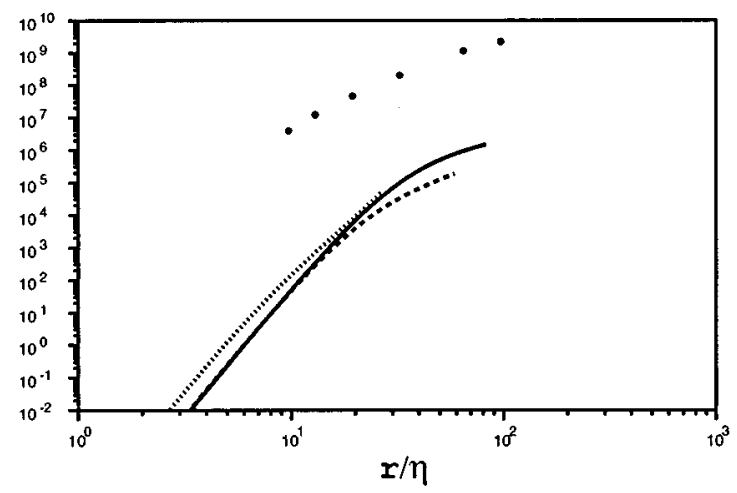

FIG. 1. Longitudinal velocity structure functions $\overline{\overline{\left(u_{p}^{\prime}-u_{p}\right)^{m}}} /(\epsilon \nu)^{m / 4}$ for the Burgers-Townsend vortex versus $r / \eta, \eta=\left(\nu^{3} / \epsilon\right)^{1 / 4}$. (a) $m=2$, (b) $m=4$, (c) $m=8$. $-\Gamma / \nu=10^{3},---\Gamma / \nu=5 \times 10^{2}, \cdots \Gamma / \nu=10^{2}$. Dots—experiment, Tabeling (Ref. 11), $R_{\lambda}=507$.

$m=8$. The odd longitudinal structure functions with $m=3$, $m=5$ and $m=7$ are displayed in Figure 2. Since these were found to be always negative we have plotted their negative values of $-\overline{\left(u_{p}^{\prime}-u_{p}\right)^{m}} /(\epsilon \nu)^{m / 4}$ in order to use log-log axes. The Tabeling data intersect the calculations but the latter show no indication of a dependence proportional to $r$.

\section{THE LUNDGREN STRETCHED-SPIRAL VORTEX}

\section{A. Velocity in the spiral}

We now turn to the stretched-spiral vortex. It is convenient to use the Lundgren transform described in $L$, which identifies an unsteady three-dimensional flow in a uniform strain with a two-dimensional motion in stretched coordinates $(\rho, \theta, \tau)$, where

$$
\rho=s e^{a t / 2}, \quad \tau=\left(e^{a t}-1\right) / a .
$$

(a)

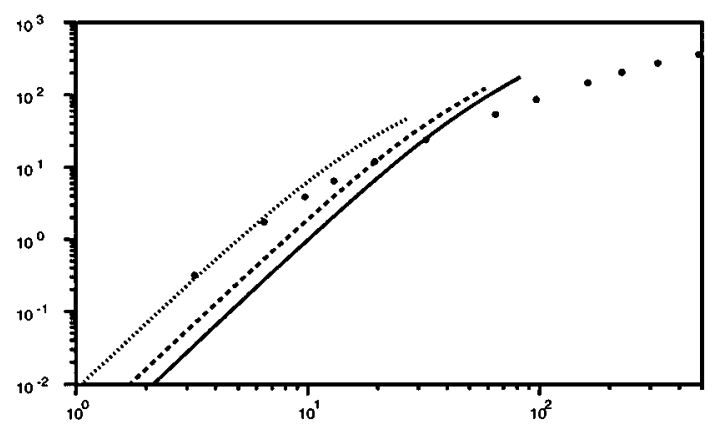

(b)

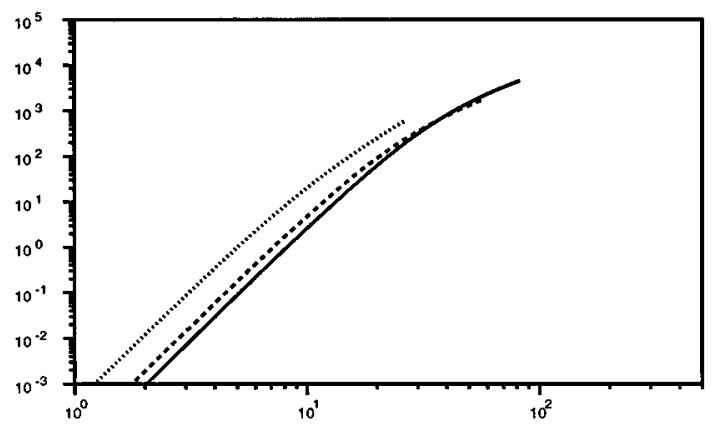

(c)

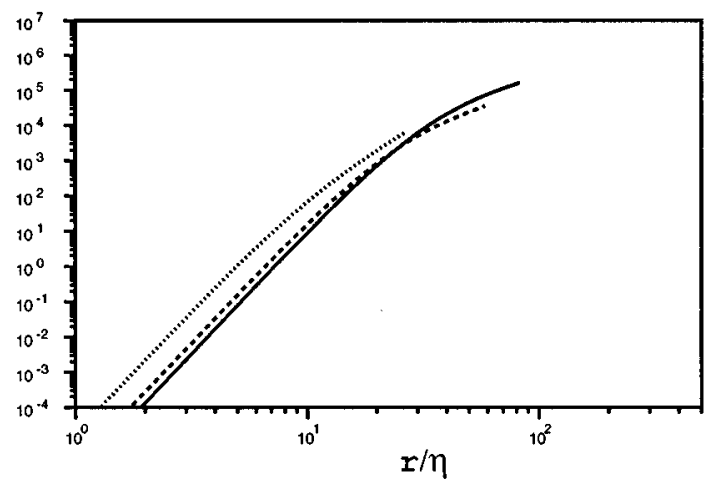

FIG. 2. Negative longitudinal structure functions $-\overline{\overline{\left(u_{p}^{\prime}-u_{p}\right)^{m}}} /(\epsilon \nu)^{m / 4}$ for the Burgers-Townsend vortex versus $r / \eta$. (a) $m=3$, (b) $m=5$, (c) $m=7$. For key, see Figure 2.

If $\hat{\boldsymbol{u}}(\rho, \theta, \tau), \hat{\boldsymbol{\omega}}(\rho, \theta, \tau)$ is a plane two-dimensional NavierStokes solution, then

$$
\begin{aligned}
& \omega(s, \theta, t)=e^{a t} \hat{\boldsymbol{\omega}}(\rho, \theta, \tau), \\
& \boldsymbol{u}(s, \theta, t)=e^{a t / 2} \hat{\boldsymbol{u}}(\rho, \theta, \tau),
\end{aligned}
$$

is a stretched vortex in the axisymmetric uniform strain (17). We can write (22) in terms of the stretched coordinates as

$$
\begin{aligned}
\overline{\overline{\left(u_{p}^{\prime}-u_{p}\right)^{m}}}= & \left\{\frac{N}{4 \pi a} \int_{T_{1}}^{T_{2}} \frac{d T}{1+T} \int_{0}^{\infty} \rho d \rho \int_{0}^{2 \pi} d \theta\right. \\
& \times \int_{0}^{\pi} \sin \alpha d \alpha \int_{0}^{2 \pi} d \beta\left[\left((1+T)^{1 / 2} \delta \hat{u}(\rho, \theta, \tau)\right.\right. \\
& \left.\left.\left.+\delta u_{s}\right)^{m}-\left(\delta u_{s}\right)^{m}\right]\right\}+b_{m}^{(s)}(r) .
\end{aligned}
$$

Here, $\delta \hat{u}(\rho, \theta, \tau)$ is $\delta u_{v}$ given by (18) with $u_{\theta}(s, \theta, t)$ replaced by $\hat{u}_{\theta}(\rho, \theta, \tau)$ and $u_{\theta}^{\prime}\left(s^{\prime}, \theta^{\prime}, t\right)$ by $\hat{u}_{\theta}^{\prime}\left(\rho^{\prime}, \theta^{\prime}, \tau\right)$, after $s^{\prime}$ and $\theta^{\prime}$ have been determined from (16). Nondimensional stretched times are defined by 


$$
a \tau=T, \quad T_{1}=a \tau_{1}, \quad T_{2}=a \tau_{2},
$$

where $T_{1}$ and $T_{2}$ refer to beginning and end times of the vortex life, respectively. For estimates of the lifetime, we take $T_{1}=1$, and from PS (25), PBS (55), we take

$$
T_{2}=C\left(\frac{a R^{2}}{4 \nu}\right)\left(\frac{\nu}{\Gamma}\right)^{2 / 3}
$$

Here, $R$ is a characteristic radius and $\Gamma$ is a characteristic circulation of the vortex. The numerical studies of PBS suggest $C \approx 10$. The quantities $\Gamma / \nu$ and $a R^{2} / 4 \nu$ are dimensionless parameters to be specified as part of the model. Further dimensionless parameters to be included later are $a^{2} \nu / \epsilon$ and $N R^{2} / a$. It remains to specify the tangential velocity $\hat{u}_{\theta}(\rho, \theta, \tau)$ so that we can calculate $\delta \hat{u}$ and close the problem. We take (based on $L$ and PBS) the vorticity field to be

$$
\hat{\omega}(\rho, \theta, \tau)=\sum_{-\infty}^{\infty} \hat{\omega}_{n}(\rho, \tau) e^{i n \theta}
$$

where

$$
\begin{aligned}
& \hat{\omega}_{n}=f(\rho) \exp \left(-i n \Omega(\rho) \tau-\nu n^{2} \Lambda^{2}(\rho) \tau^{3} / 3\right) \\
& \hat{\omega}_{0}=f(\rho)+g(\rho) .
\end{aligned}
$$

Here, $e^{a t} f(\rho)$ will be the $\theta$-averaged vorticity for the spiral, and $e^{a t} g(\rho)$ will be an axisymmetric background vorticity field. The angular velocity $\Omega$ is given by

$$
\frac{d}{d \rho} \rho^{2} \Omega(\rho)=\rho(g(\rho)+f(\rho)) \quad \text { and } \Lambda(\rho) \equiv d \Omega / d \rho \text {. }
$$

For $f(\rho)$, we have in mind that the asymmetric part of the vorticity distribution comes from the roll-up of a vortex sheet resembling a Kaden spiral and take

$$
\begin{aligned}
& f(\rho)=\frac{\Gamma}{R^{2}}\left(\frac{\rho}{R}\right)^{\alpha} \frac{e^{-(\rho / R)^{2}}}{\pi \Gamma[1+(\alpha / 2)]}, \\
& g(\rho)=-\frac{\Gamma}{\pi R^{2}}\left(\frac{\rho}{R}\right)^{2} e^{-(\rho / R)^{2}} .
\end{aligned}
$$

The coefficients have been adjusted so that

$$
\int_{0}^{\infty} \rho(f(\rho)+g(\rho)) d \rho=0
$$

the total circulation of the vortex structure is then zero. Note also that the amplitudes of the Fourier coefficients of the vorticity, Equation (53), are taken to be independent of $n$; the vorticity is then in the form of a rolled up vortex spiral sheet in the limit $\nu \rightarrow 0$. We shall call (56) and (57) the "standard model.' The value $\alpha=-1 / 2$ is the Kaden spiral. Generally, $\alpha>-1$ is required to make the circulation finite. The tangential velocity $\hat{u}_{\theta}(\rho, \theta, \tau)$ is taken to be

$$
\begin{aligned}
\hat{u}_{\theta}(\rho, \theta, \tau)= & \rho \Omega(\rho)-\frac{2 f(\rho)}{\tau \Lambda(\rho)} \sum_{1}^{\infty} \frac{1}{n} \sin n(\theta-\Omega \tau) \\
& \times e^{-\nu \Lambda^{2} \tau^{3} n^{2} / 3} .
\end{aligned}
$$

To leading order in $\tau^{-1}$, it can be verified that when $\nu=0$,

$$
\hat{\omega}(\rho, \theta, \tau)=\frac{1}{\rho} \frac{\partial}{\partial \rho}\left(\rho \hat{u}_{\theta}(\rho, \theta, \tau)\right)
$$

\section{B. Calculation of structure functions by Monte Carlo integration}

We nondimensionalize velocities on $a R$, lengths on $R$, and put $\tau=T / a$. We define $\xi=\rho / R$. A $\sim$ is used for dimensionless functions. We write

$$
\begin{aligned}
& \hat{u}_{\theta}(\rho, \theta, \tau)=a R \widetilde{u}(\xi, \theta, T), \\
& f(\rho)=\frac{\Gamma}{R^{2}} \widetilde{f}(\xi), \quad g(\rho)=\frac{\Gamma}{R^{2}} \widetilde{g}(\xi), \\
& \Omega(\rho)=\frac{\Gamma}{R^{2}} \widetilde{\Omega}(\xi), \quad \Gamma(\rho)=\frac{\Gamma}{R^{3}} \widetilde{\Lambda}(\xi) .
\end{aligned}
$$

The contribution from the spiral is from (59)

$$
\begin{aligned}
\tilde{u}= & -2 \frac{\tilde{f}}{T \widetilde{\Lambda}} \sum_{1}^{\infty} \sin n\left(\theta-\frac{1}{4}\left(\frac{\Gamma}{\nu}\right)\left(\frac{4 \nu}{a R^{2}}\right) \widetilde{\Omega} T\right) \\
& \times \exp \left[-\frac{1}{192} \widetilde{\Lambda}^{2} T^{3}\left(\frac{\Gamma}{\nu}\right)^{2}\left(\frac{4 \nu}{a R^{2}}\right)^{3} n^{2}\right]+\frac{1}{4}\left(\frac{\Gamma}{\nu}\right) \\
& \times\left(\frac{4 \nu}{a R^{2}}\right) \xi \widetilde{\Omega}(\xi) .
\end{aligned}
$$

Then we can write (49) as

$$
\begin{aligned}
\overline{\overline{\left(u_{p}^{\prime}-u_{p}\right)^{m}}}= & \frac{1}{4 \pi}\left(\frac{N R^{2}}{a}\right)(a R)^{m} \int_{T_{1}}^{T_{2}}(1+T)^{m / 2-1} d T \int_{0}^{\infty} \xi d \xi \int_{0}^{2 \pi} d \theta \int_{0}^{\pi} \sin \alpha d \alpha \int_{0}^{2 \pi} d \beta \\
& \times\left\{\left[\delta \tilde{u}+\frac{\tilde{r}}{(1+T)^{1 / 2}}\left(\cos ^{2} \alpha-\frac{1}{2} \sin ^{2} \alpha\right)\right]^{m}-\left[\frac{\tilde{r}}{(1+T)^{1 / 2}}\left(\cos ^{2} \alpha-\frac{1}{2} \sin ^{2} \alpha\right)\right]^{m}\right\}+b_{m}^{(s)}(r),
\end{aligned}
$$

where $\delta \tilde{u}=\delta \hat{u} / a R$ and we repeat that $\delta \hat{u}$ is given by (18) with $u_{\theta}(s, \theta, t)$ replaced by $\hat{u}_{\theta}(\rho, \theta, \tau)$ etc., and is a function of $\tilde{r}=r / R$ by virtue of (16) and (47). See (68) and (69) below. 
We make the structure function dimensionless by dividing by $(\epsilon \nu)^{m / 4}$. This gives

$$
\begin{aligned}
\overline{\frac{\left(u_{p}^{\prime}-u_{p}\right)^{m}}{(\epsilon \nu)^{m / 4}}=} & \frac{2^{m-2}}{\pi}\left(\frac{N R^{2}}{a}\right)\left(\frac{a R^{2}}{4 \nu}\right)^{m / 2}\left(\frac{a^{2} \nu}{\epsilon}\right)^{m / 4} \int_{T_{1}}^{T_{2}}(1+T)^{m / 2-1} d T \int_{0}^{\infty} \xi d \xi \int_{0}^{2 \pi} d \theta \int_{0}^{\pi} \sin \alpha d \alpha \\
& \times \int_{0}^{2 \pi} d \beta\left\{\left[\delta \tilde{u}+\frac{\tilde{r}}{(1+T)^{1 / 2}}\left(\cos ^{2} \alpha-\frac{1}{2} \sin ^{2} \alpha\right)\right]^{m}-\left[\frac{\tilde{r}}{(1+T)^{1 / 2}}\left(\cos ^{2} \alpha-\frac{1}{2} \sin ^{2} \alpha\right)\right]^{m}\right\} \\
& +\tilde{r}^{m} k_{m} 2^{m}\left(\frac{a R^{2}}{4 \nu}\right)^{m / 2}\left(\frac{a^{2} \nu}{\epsilon}\right)^{m / 4}
\end{aligned}
$$

Evaluation of the integral is carried out by the Monte Carlo technique as follows.

(1) Pick values of the four dimensionless model parameters $a R^{2} / 4 \nu, a^{2} \nu / \epsilon, N R^{2} / a, \Gamma / \nu$. See PBS Table III for suitable values.

(2) Choose a value of the separation made dimensionless by the Kolmogorov length $\eta=\nu^{3} / \epsilon$ and calculate the corresponding value of $\tilde{r}$, i.e.

$$
\tilde{r}=\frac{1}{2}\left(\frac{4 \nu}{a R^{2}}\right)^{1 / 2}\left(\frac{a^{2} \nu}{\epsilon}\right)^{1 / 4}\left(\frac{r}{\eta}\right) .
$$

(3) Calculate $T_{2}$ from (51).

(4) Choose random values of $T, \xi, \theta, \alpha, \beta$ uniformly distributed in the five-dimensional rectangle $T_{1}<T<T_{2}$, $0<\xi<\xi_{\max }, 0<\theta<2 \pi, 0<\alpha<\pi, 0<\beta<2 \pi$.

(5) Use Equation (64) to evaluate $\widetilde{u}(\xi, \theta, T)$.

(6) Use the values of $\xi$ and $T$ in (50) to calculate $\tilde{s}=s / R=\xi /(1+T)^{1 / 2}$.

(7) Use (16), after dividing by $R$, to give

$$
\begin{aligned}
\tilde{s}^{\prime}=s^{\prime} / R= & {\left[(\tilde{s} \cos \theta+\widetilde{r} \sin \alpha \cos \beta)^{2}+(\tilde{s} \sin \theta\right.} \\
& \left.+\widetilde{r} \sin \alpha \sin \beta)^{2}\right]^{1 / 2}
\end{aligned}
$$

and

$$
\theta^{\prime}=\tan ^{-1} \frac{\widetilde{s} \sin \theta+\widetilde{r} \sin \alpha \sin \beta}{\widetilde{s} \cos \theta+\widetilde{r} \sin \alpha \cos \beta} .
$$

(8) From (47),

$$
\xi^{\prime}=\widetilde{s}^{\prime}(1+T)^{1 / 2} \text {. }
$$

(9) Use (64) with the values of $\xi^{\prime}, \theta^{\prime}$ and $T$ to determine $\tilde{u}^{\prime}\left(\xi^{\prime}, \theta^{\prime}, T\right)$.

(10) Substitute into (18), replacing $u_{\theta}$ by $\tilde{u}_{\theta}$, etc. to give $\delta \tilde{u}$.

(11) Go to (4) and repeat until satisfactory convergence of the integral is obtained and the result is independent of $\xi_{\max }$.

Numerical calculations were done with $M=2 \times 10^{6}$ integration points for each value of $r / \eta$. In the detailed calculations, advantage was taken of the symmetry of the integrand about $\alpha=\pi / 2$. A cutoff in the $\xi$-integration at $\xi=6.26$ was used. Independent codes were written by both authors and results were compared for specific values of the separation. In addition, some calculations were done with smaller $M$ using several random number generators and the results were found to agree to within the expected statistical error of order $M^{-1 / 2}$. The specific results presented in Figures 4 and 5 were done with values

$$
\frac{\Gamma}{\nu}=10^{3}, \quad \frac{N R^{2}}{a}=0.147, \quad \frac{a R^{2}}{4 \nu}=49, \quad \frac{a^{2} \nu}{\epsilon}=0.0149,
$$

chosen from Table III of PBS to give $R_{\lambda} \approx 500$ to match the experiments of Tabeling. ${ }^{11}$ Figure 3 shows a calculation of the negative third-order structure function compared with the results of Tabeling ${ }^{11}$ at $R_{\lambda}=507$. Also shown is the result of a small $r / \eta$ approximation

$$
\frac{\overline{\left(u_{p}^{\prime}-u_{p}\right)^{m}}}{(\epsilon \nu)^{m / 4}}=\frac{\overline{\overline{(\partial u / \partial x)^{m}}}}{\left(\overline{\overline{(\partial u / \partial x)^{2}}}\right)^{m / 2}}\left(\frac{1}{15}\right)^{m / 2}\left(\frac{r}{\eta}\right)^{m},
$$

obtained using $m=3$ and the estimate of the cubic velocity derivative from PBS. The Monte Carlo results were found to change sign near $r / \eta \approx 20$ and again near $r / \eta \approx 120$. While numerically the calculations are within a factor of about 2 of the data, they do not show any definite range of proportionality to $r$, nor do they show any domain of overlap with the estimate from the cubic longitudinal velocity derivative. We stress that we use no additional parameters other than those used by PBS. Also shown in Figure 3 is the calculation for the Burgers-Townsend model for $\Gamma / \nu=1000$ reproduced from Figure 2. Over the range of $\tilde{r}$ displayed, which includes small separation, the spiral-vortex results are marginally superior to those of the Townsend-Burgers model.

In Appendix B we discuss an alternative method for the calculation of $B_{3}(r)$ to that used presently. It is argued from kinematics and from the spectral form of the KármańHowarth equation that in the inertial subrange $B_{3}(r)$ can be viewed as a dual large-scale/small-scale property. This is consistent with its special dynamical role in the transfer of energy from large to small scales. From the standpoint of the Fourier integral, it is shown in Appendix B that the Kolmogorov form (14) in the inertial range may be obtained either from the small wave number, energy containing range, or equivalently from the large wave number dissipative range, but, paradoxically, not from the inertial range of wave numbers where the spectral equivalent of $B_{3}(r)$, the function $F_{3}(k)$ [see (B3) and (B4)], is effectively zero when $\nu \rightarrow 0$. In the present Monte Carlo calculations, on the one hand, our estimate of the effect of the large scales is fairly crude, entering only though our inclusion of the background velocity 


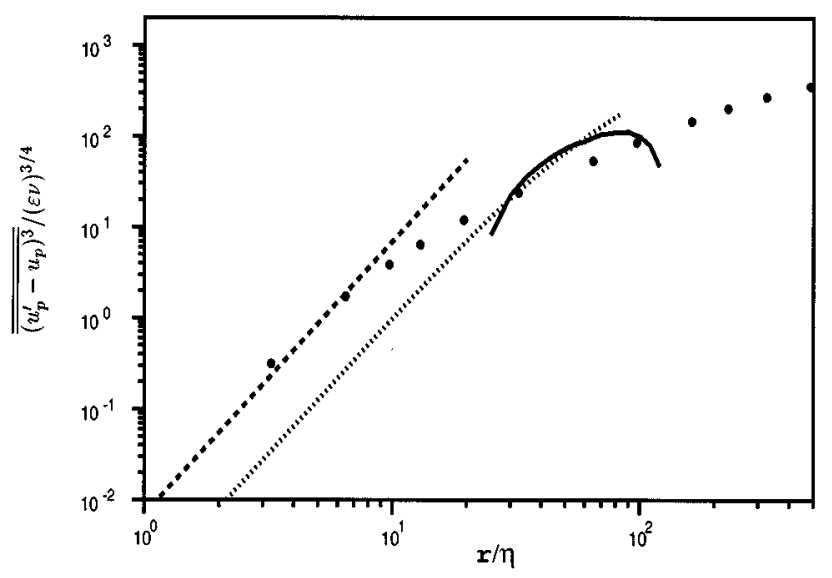

FIG. 3. Negative third-order longitudinal velocity structure functions $\overline{\overline{\left(u_{p}^{\prime}-u_{p}\right)^{3}}} /(\epsilon \nu)^{3 / 4}$. - stretched-spiral vortex, Monte-Carlo, $R_{\lambda}=500$. --small $r / \eta$ approximation using $\overline{\overline{(\partial u / \partial x)^{3}}}$ from PBS. $\cdots$ Townsend-Burgers model. Dots—experiment, Tabeling (Ref. 11), $R_{\lambda}=507$.

field, which in the model is supposed to represent the stretching effect of the larger scales, and in the relation between the model parameters and the large scale quantities proposed in Sec. V B of PBS. On the other hand, as argued below, the present velocity field is in error at small separations. These deficiencies of the present model will contribute to the discrepancies between calculation and experiment of Figure 3 .

In Figure 4 we show the even velocity structure functions for $m=2, m=4, m=6$ and $m=8$ compared with Tabeling's $\mathrm{s}^{11}$ data for these quantities. We display our results only in the range $10 \leqslant r / \eta \leqslant 200$. The reason for the lower cutoff is that our expression for the velocity difference obtained from (59) is not accurate at small separation $r$ when $\nu \neq 0$. This is because (60) is not uniformly valid in both $\tau$ and $\rho$ in this case, which can be seen by substituting (59) into (60) and noting the nonuniformities produced by the presence of the exponential in (59), when $\nu \neq 0$. The upper limit is imposed by the requirement that, since the PBS model

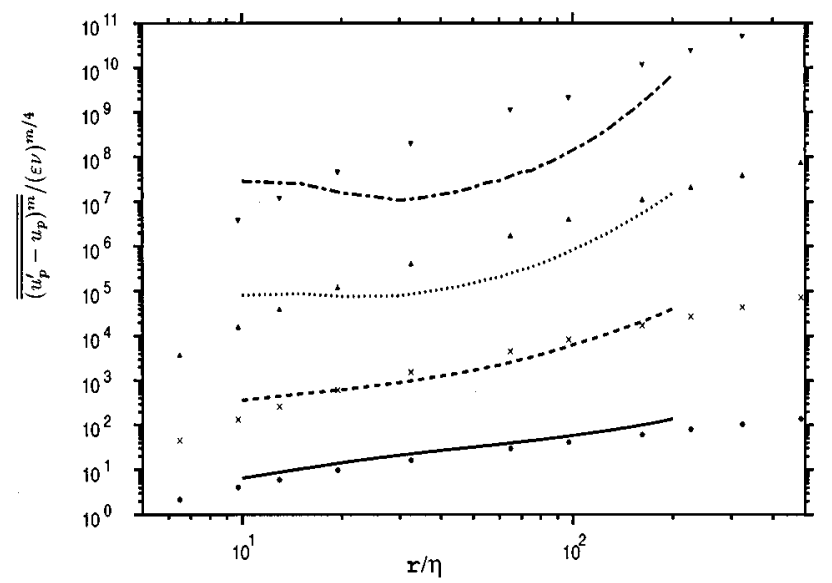

FIG. 4. Longitudinal velocity structure functions $\overline{\overline{\left(u_{p}^{\prime}-u_{p}\right)^{m}}} /(\epsilon \nu)^{m / 4}$ for the stretched-spiral vortex versus $r / \eta$, Monte Carlo, $R_{\lambda}=500$. $-m=2$, --$m=4, \cdots m=6, \cdots m=8$ : Symbols-experiment, Tabeling (Ref. 11), $R_{\lambda}=507$. Circles $m=2$, crosses $m=4$, up-triangles $m=6$, down-triangles $m=8$.

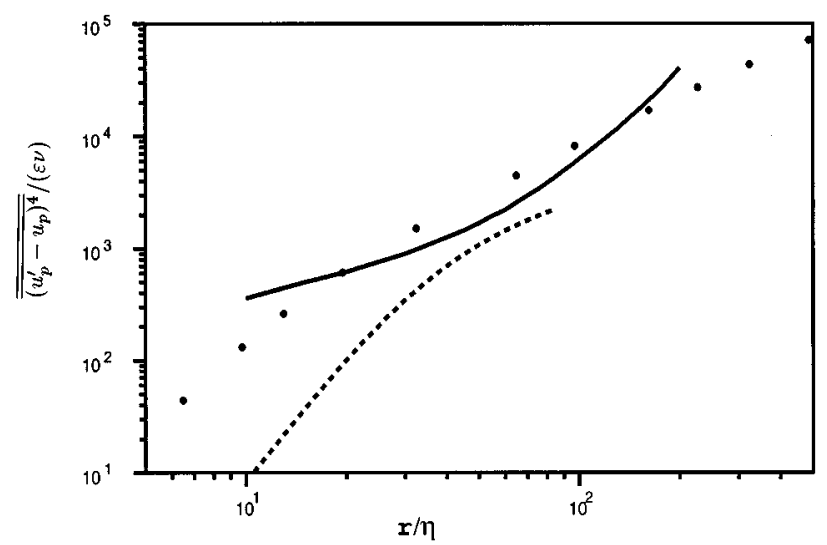

FIG. 5. Longitudinal fourth-order velocity structure function $\overline{\overline{\left(u_{p}^{\prime}-u_{p}\right)^{4}}} /$ $(\epsilon \nu)$. - stretched-spiral vortex, $R_{\lambda}=500$. --- Townsend-Burgers model. Circles-experiment, Tabeling (Ref. 11), $R_{\lambda}=507$.

assumes closely packed vortices, the separation should not exceed a dimension of order the vortex diameter $2 R$. For separations larger than this, the two points would have a substantial probability of feeling the velocity-induction of more than one vortex, in contradiction of the model ansatz based on the field of a single vortex.

The agreement with the data in Figure 4 is reasonable for the second and fourth order, and although it degrades at the higher orders, the spiral-vortex results are always superior to those of the Burgers-Townsend calculations of Figure 1. This is illustrated in Figure 5 which compares the spiralvortex and the Burgers-Townsend results for $m=4$. Anselmet et al. ${ }^{13}$ measure structure functions up to high order in duct and jet flows. Figure 6 shows a comparison with their measurements in a jet at $R_{\lambda}=852$. The separation is fixed at $r / \eta \approx 50$ and the results are plotted against order $m$ for $m$ even. The calculations and the data diverge at high order. In assessing these results it should be kept in mind that we are attempting a direct calculation of the numerical values. The dimensionless numbers are very large. We are also uncertain of the accuracy of the experiments at these very high orders.

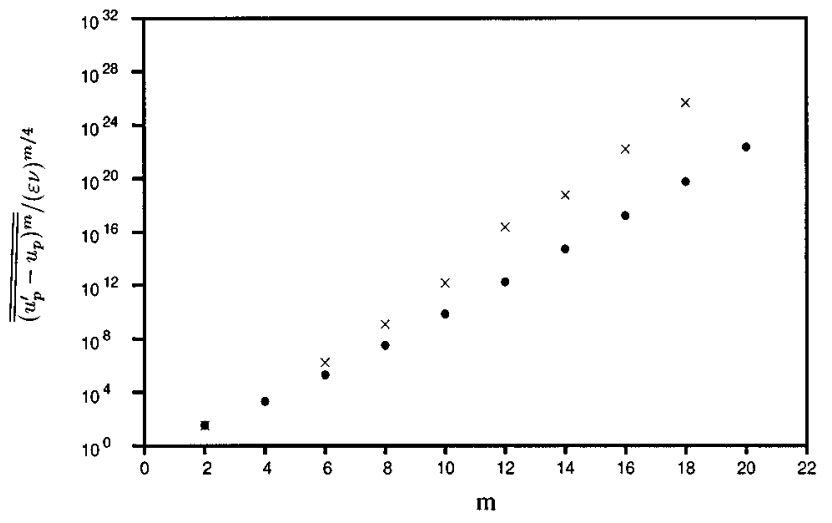

FIG. 6. Longitudinal velocity structure functions $\overline{\overline{\left(u_{p}^{\prime}-u_{p}\right)^{m}}} /(\epsilon \nu)^{m / 4}$ for the stretched-spiral vortex versus order $m$ for $m$ even. $R_{\lambda}=850, r / \eta=50$. Circles are Monte Carlo calculation for stretched-spiral vortex. Crosses, experiment, Anselmet et al. (Ref. 13) $R_{\lambda}=852$. 


\section{CONCLUSIONS}

In this paper, we have explored the predictions of two point, multi-order longitudinal velocity structure functions that can be made from the Townsend and Lundgren vortex models of isotropic turbulence. The models differ in the choice of underlying vorticity field. The Townsend model consists of steady axisymmetric Burgers vortices and the Lundgren model is based on asymmetric stretched unsteady spiral vortices. The second- and third-order structure functions for the Townsend model can be shown to satisfy the Kármán-Howarth equation, while the Lundgren model produces $r^{2 / 3}$ scaling for the second-order velocity structure function in a way independent of the detailed spiral structure and of dimensional constraints. The predictions of the spiral model for even order structure functions up to order six (Figure 4) is considered satisfactory for the spiral model and superior to the equivalent results for the Burgers-Townsend model. The third-order structure function (Figure 3) shows only fair agreement with the Kolmogorov result (14), for separations much larger than the Kolmogorov length, for both models. For the spiral vortex, on the one hand, errors are introduced by nonuniformities in the domain of validity of Equation (59) for the azimuthal velocity. These may be compounded by the cancellations inherent in the calculation of odd-order structure functions. If this is the case, a more refined calculation may give better results. On the other hand we have been unable to demonstrate that the spiral dynamics (a) approximately satisfies the Kármán-Howarth equation and (b) that even if it does, the dissipation and transfer terms are dominant over the unsteady and viscous term as in the Kolmogorov argument leading from (13) to (14). Since the spiral evolution satisfies the Navier-Stokes equations approximately, we guess that (a) is true to some order of approximation but can make no estimate of the validity of (b).

Higher-order moments may be sensitive to the detailed spiral structure, and further work is needed in order to improve on the present model of the velocity field from the given vorticity field. The current agreement between the model predictions and experiment for the fourth- and sixthorder structure functions for the spiral vortex is promising. Finally we remark that the present model could be used to calculate structure functions for turbulence properties other than the longitudinal velocity difference, for example the structure functions of the pressure, the dissipation, the enstrophy or the normal velocity difference. With some modifications, the Monte Carlo method could also be applied to the calculation of multi-point structure functions.

\section{ACKNOWLEDGMENTS}

The authors wish to thank Patrick Tabeling for supplying unpublished measurements of longitudinal velocity structure functions. D.I.P. was partially supported by NSF Grant No. CTS-9311811 and P.G.S. was partially supported by the Department of Energy under Grant No. DE-FG03-89ER25073. We thank Dan Meiron and Donal Gallagher for helping with the asymptotic expansion of (34) for large $r$.

\section{APPENDIX A: $d u / d x$ MOMENTS FOR THE} TOWNSEND MODEL

Here we obtain moments of the longitudinal velocityderivative for the Townsend-Burgers model. The orientation-averaged moments are given by PS equations (52a)52(j). We consider $m-2, \ldots, 8$ for which

$\begin{aligned}\left\langle\left(\frac{\partial u}{\partial x}\right)^{2}\right\rangle & =\frac{2\left(e_{1}^{2}+e_{2}^{2}+e_{3}^{2}\right)}{15}, \\ \left\langle\left(\frac{\partial u}{\partial x}\right)^{3}\right\rangle & =\frac{8\left(e_{1} e_{2} e_{3}\right)}{35}, \\ \left\langle\left(\frac{\partial u}{\partial x}\right)^{4}\right\rangle & =\frac{8\left(e_{1}^{4}+e_{2}^{4}+e_{3}^{4}\right)}{105},\end{aligned}$

$\left\langle\left(\frac{\partial u}{\partial x}\right)^{5}\right\rangle=\frac{32 e_{1} e_{2} e_{3}\left(e_{1}^{2}+e_{2}^{2}+e_{3}^{2}\right)}{231}$,

$\left\langle\left(\frac{\partial u}{\partial x}\right)^{6}\right\rangle=\frac{32\left[5\left(e_{1}^{6}+e_{2}^{6}+e_{3}^{6}\right)-3 e_{1}^{2} e_{2}^{2} e_{3}^{2}\right]}{3003}$,

$\left\langle\left(\frac{\partial u}{\partial x}\right)^{7}\right\rangle=\frac{32 e_{1} e_{2} e_{3}\left(e_{1}^{2}+e_{2}^{2}+e_{3}^{2}\right)^{2}}{429}$,

$\left\langle\left(\frac{\partial u}{\partial x}\right)^{8}\right\rangle=\frac{\left(e_{1}^{2}+e_{2}^{2}+e_{3}^{2}\right)\left[448\left(e_{1}^{6}+e_{2}^{6}+e_{3}^{6}\right)+1728 e_{1}^{2} e_{2}^{2} e_{3}^{2}\right]}{21879}$,

where $\left(e_{1}, e_{2}, e_{3}\right)$ are the principal rates of strain in vortexfixed axes. For the Burgers vortex these are given by

$$
e_{1}=-\frac{1}{2} a+e_{r \theta}, \quad e_{2}=-\frac{1}{2} a-e_{r \theta}, \quad e_{3}=a,
$$

where

$$
e_{r \theta}=\frac{1}{2} \frac{\partial u_{\theta}}{\partial r}-\frac{u_{\theta}}{2 r}
$$

with $u_{\theta}$ given by (26). The velocity derivative moments were calculated by use of (26) in (A3), which is then used in (A1a)-(A1f), followed by application of the formula

$$
\begin{aligned}
\overline{\overline{\left(\frac{\partial u}{\partial x}\right)^{n}}=} & 2 \pi N \tau_{2} \int_{0}^{\infty}\left(D_{n}\left(e_{1}, e_{2}, e_{3}\right)\right. \\
& \left.-D_{n}(-a / 2,-a / 2, a)\right) r d r+a^{m} k_{m},
\end{aligned}
$$

where $D_{n}\left(e_{1}, e_{2}, e_{3}\right)=\left\langle(\partial u / \partial x)^{n}\right\rangle$ from (A1a)-(A1f) and where we have added and subtracted the contribution due to the pure strain, as for (21), in order to produce a convergent integral. The evaluation of the integrals in (A4) is tedious but straightforward. When the $\overline{\overline{(\partial u / \partial x)^{n}}}$ are normalized against

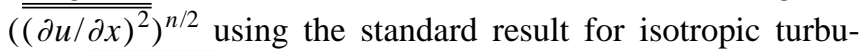
lence $\overline{\overline{(\partial u / \partial x)^{2}}}=\epsilon / 15 \nu$ we obtain,

$$
\frac{\overline{\overline{(\partial u / \partial x)^{2}}}}{\overline{\left(\overline{(\partial u / \partial x)^{2}}\right)^{1}}}=\frac{a^{2} \nu}{\epsilon}\left[\frac{1}{8 \pi} \frac{N \tau_{2} \nu}{a}\left(\frac{\Gamma}{\nu}\right)^{2}+3\right],
$$


$\frac{\overline{\overline{\partial u / \partial x)^{3}}}}{\left.\overline{\overline{\left((\partial u / \partial x)^{2}\right.}}\right)^{3 / 2}}=\frac{15^{3 / 2}}{35}\left(\frac{a^{2} \nu}{\epsilon}\right)^{3 / 2}\left[-\frac{1}{4 \pi} \frac{N \tau_{2} \nu}{a}\left(\frac{\Gamma}{\nu}\right)^{2}+2\right]$

(A5b)

$\begin{aligned} \frac{\overline{\overline{(\partial u / \partial x)^{4}}}}{\overline{\left.\overline{\left((\partial u / \partial x)^{2}\right.}\right)^{2}}=} & \left(\frac{a^{2} \nu}{\epsilon}\right)^{3 / 2}\left[\frac{N \tau_{2} \nu}{a}\left(G_{44}\left(\frac{\Gamma}{\nu}\right)^{4}+G_{42}\left(\frac{\Gamma}{\nu}\right)^{2}\right)\right. \\ & \left.+G_{40}\right]\end{aligned}$

$\frac{\overline{\overline{(\partial u / \partial x)^{5}}}}{\overline{\left.\overline{\left((\partial u / \partial x)^{2}\right.}\right)^{5 / 2}}}=\left(\frac{a^{2} \nu}{\epsilon}\right)^{5 / 2}\left[\frac{N \tau_{2} \nu}{a}\left(G_{54}\left(\frac{\Gamma}{\nu}\right)^{4}+G_{52}\left(\frac{\Gamma}{\nu}\right)^{2}\right)\right.$

$\left.+G_{50}\right]$

$$
\begin{aligned}
& \frac{\overline{\overline{(\partial u / \partial x)^{6}}}}{\overline{\left(\overline{(\partial u / \partial x)^{2}}\right)^{3}}}=\left(\frac{a^{2} \nu}{\epsilon}\right)^{3}\left[\frac { N \tau _ { 2 } \nu } { a } \left(G_{66}\left(\frac{\Gamma}{\nu}\right)^{6}+G_{64}\left(\frac{\Gamma}{\nu}\right)^{4}\right.\right. \\
&\left.\left.+G_{62}\left(\frac{\Gamma}{\nu}\right)^{2}\right)+G_{60}\right]
\end{aligned}
$$

$$
\begin{aligned}
& \frac{\overline{\overline{(\partial u / \partial x)^{7}}}}{\overline{\overline{\left((\partial u / \partial x)^{2}\right)^{7 / 2}}}}=\left(\frac{a^{2} \nu}{\epsilon}\right)^{7 / 2}\left[\frac { N \tau _ { 2 } \nu } { a } \left(G_{76}\left(\frac{\Gamma}{\nu}\right)^{6}+G_{74}\left(\frac{\Gamma}{\nu}\right)^{4}\right.\right. \\
& \left.\left.+G_{72}\left(\frac{\Gamma}{\nu}\right)^{2}\right)+G_{70}\right]
\end{aligned}
$$

$$
\begin{aligned}
\frac{\overline{\overline{(\partial u / \partial x)^{8}}}}{\overline{\left.\overline{\left((\partial u / \partial x)^{2}\right.}\right)^{8}}}= & \left(\frac{a^{2} \nu}{\epsilon}\right)^{4}\left[\frac { N \tau _ { 2 } \nu } { a } \left(G_{88}\left(\frac{\Gamma}{\nu}\right)^{8}+G_{86}\left(\frac{\Gamma}{\nu}\right)^{6}\right.\right. \\
& \left.\left.+G_{84}\left(\frac{\Gamma}{\nu}\right)^{4}+G_{82}\left(\frac{\Gamma}{\nu}\right)^{2}\right)+G_{80}\right],
\end{aligned}
$$

where, from the integrals we find

$$
\begin{aligned}
& G_{40}=1.929 \times 10^{1}, \quad G_{42}=5.116 \times 10^{-1}, \\
& G_{44}=2.534 \times 10^{-5}, \\
& G_{50}=4.527 \times 10^{1}, \quad G_{52}=1.201 \times 10^{0}, \\
& G_{54}=1.784 \times 10^{-4}, \\
& G_{60}=1.787 \times 10^{2}, \quad G_{62}=3.890 \times 10^{0}, \\
& G_{64}=9.171 \times 10^{-4}, \quad G_{66}=2.872 \times 10^{-8}, \\
& G_{70}=5.484 \times 10^{2}, \quad G_{72}=7.274 \times 10^{0}, \\
& G_{74}=3.603 \times 10^{-3}, \quad G_{76}=3.114 \times 10^{-7}, \\
& G_{80}=1.978 \times 10^{3}, \quad G_{82}=2.541 \times 10^{1}, \\
& G_{84}=1.297 \times 10^{-2}, \quad G_{86}=2.128 \times 10^{-6}, \\
& G_{88}=3.934 \times 10^{-11} .
\end{aligned}
$$

The right-hand sides of (A5a)-(A5e) depend on three dimensionless parameters. This number can be reduced to two independent parameters by use of (43). The right-hand side of (A5a) is then unity as required. As in Sec. III we choose $N \tau_{2} \nu / a=1 /(4 \pi)$. Values of the velocity-derivative moments
$F_{2 p}$ and $S_{2 p+1}$ defined as in (1) are given, for the BurgersTownsend model, in Table I for three values of $\Gamma / \nu$. The results show no increase of $\left|\overline{\overline{(\partial u / \partial x)^{n}}}\right|$ with order. Further, they are well out of range of the experimental measurements of Tabeling ${ }^{11}$ and the numerical results of Jiménéz et al. ${ }^{14}$

We also considered the use of (A5a)-(A5g) to test the hypothesis that the tubes of extremely high vorticity, the socalled "worms,", which have been observed in $\mathrm{DNS}^{14}$ and experiment can alone account for the higher-order longitudinal velocity derivative moments. We were, however, unable to settle upon a satisfactory means of fixing the three dimensionless groupings to estimate parameters in this case, and so we leave this problem to the reader, noting that (A5a)-(A5f) give sufficient information to test various different scenarios. We note that owing to the products of powers of large $(\Gamma / \nu)$ and small $\left(a^{2} \nu / \epsilon\right)$ quantities in (A5a)-(A5g), there is strong sensitivity to numerical values. A wide range of values can be obtained for different choices of the parameters.

\section{APPENDIX B: THIRD-ORDER STRUCTURE FUNCTION}

Here we discuss a possible alternative approach to calculation of the third-order structure function, to that given in the text. The contribution to $b_{3}(\tau)$ from the vortex alone can be expressed in terms of integral over the velocity field of the vortex. We define the third-order two point velocity correlation as

$$
B_{i j, l}(\mathbf{r})=\overline{\overline{u_{i}(\mathbf{x}) u_{j}(\mathbf{x}) u_{l}(\mathbf{x}+\mathbf{r})}} .
$$

For isotropic turbulence the contraction $B_{l 3, l}(\mathbf{r})$ can be written in terms of $B_{3}(\rho)$ as (see Monin and Yaglom, ${ }^{15}$ p. 71)

$$
B_{l 3, l}(\mathbf{r})=\frac{r_{3}}{6 r}\left(2 B_{3}(r)+\frac{r}{2} \frac{\partial B_{3}(r)}{\partial r}\right),
$$

where $r_{3}$ is the three-component of the separation in laboratory coordinates and $r=|\mathbf{r}|=|\boldsymbol{r}|$. Now $B_{3}(r)$ can be expressed in terms of the scalar function of wave number $k, F_{3}(k)$, as [Monin and Yaglom, ${ }^{15}$ Equation (12.141)]

$$
\begin{aligned}
\frac{B_{3}(r)}{6}= & 16 \pi \int_{0}^{\infty}\left(\frac{\sin (k r)}{k^{2} r^{2}}+\frac{3 \cos (k r)}{k^{3} r^{3}}\right. \\
& \left.-\frac{3 \sin (k r)}{k^{4} r^{4}}\right) k^{2} F_{3}(k) d k .
\end{aligned}
$$

Substituting (B3) into (B2) gives

$$
B_{l 3, l}(\mathbf{r})=8 \pi \frac{r_{3}}{r} \int_{0}^{\infty}\left(\frac{\cos (k r)}{k r}-\frac{\sin (k r)}{k^{2} r^{2}}\right) k^{2} F_{3}(k) d k .
$$

We now express $B_{l 3, l}(\mathbf{r})$ in terms of velocity components in the frame of the vortex. The laboratory axes are $\left(r_{1}, r_{2}, r_{3}\right)$ fixed in space, and coordinates $\left(r_{1}, r_{2}, r_{3}\right)$ are vortex-fixed axes with $r_{1}, r_{2}$, lying in the plane of the cross section. We denote by $E_{i j}$ the rotation matrix describing transformations from laboratory to vortex coordinates. The components of $E_{i j}$ are expressible in terms of the Euler angles, $\alpha, \beta, \gamma$ describing rotation of the $\left(r_{1}, r_{2}, r_{3}\right)$ axes to the $\left(r_{1}, r_{2}, r_{3}\right)$ axes, where in this appendix $\alpha$ is co-latitude (angle between the $r_{3}$ and $r_{3}$ axes), $\beta$ is longitude and $\gamma$ is spin about the 
$r_{3}$-axis. We denote velocity components in laboratory axes by $u_{i}$ and in vortex axes by $u_{i}$. The quantity $B_{l 3, l}$ can be expressed as

$$
B_{l 3, l}(\mathbf{r})=\overline{\overline{u_{1} u_{3} u_{1}^{\prime}+u_{2} u_{3} u_{2}^{\prime}+u_{3} u_{3} u_{3}^{\prime}}},
$$

where $u_{i}=u_{i}(\mathbf{r})$ and $u_{i}^{\prime}=u_{i}(\mathbf{x}+\mathbf{r})$ are the velocity components at two points. Transforming to vortex axes and using that, for the velocity field of the vortex alone, $u_{3}=u_{3}^{\prime}=0$, we obtain

$$
\begin{aligned}
B_{l 3, l}(\mathbf{r}) & =\overline{\overline{E_{13}\left(u_{1} u_{1} u_{1}^{\prime}+u_{1} u_{2} u_{2}^{\prime}\right)+E_{23}\left(u_{1} u_{2} u_{1}^{\prime}+u_{2} u_{2} u_{2}^{\prime}\right)}} \\
& \equiv B_{l 3, l}^{(1)}+B_{l 3, l}^{(2)}+B_{l 3, l}^{(3)}+B_{l 3, l}^{(4)} .
\end{aligned}
$$

The quantities $B_{l 3, l}^{(p)}, p=1,4$ can be expressed in terms of the Fourier transforms of $u_{i}$ and of the products $u_{i} u_{j}$ by following the steps leading to equation (38) of Pullin and Saffman. ${ }^{16}$ This gives, for $B_{l 3, l}^{(1)}$

$$
\begin{aligned}
B_{l 3, l}^{(1)}(\mathbf{r})= & \frac{N}{2} \int_{t_{1}}^{t_{2}} e^{a t} d t \int_{-\infty}^{\infty} \int_{-\infty}^{\infty} \int_{0}^{\pi} \int_{0}^{2 \pi} \int_{0}^{2 \pi} E_{13} \\
& \times \hat{U}_{11}\left(k_{1}, k_{2}, t\right) \hat{U}_{1}^{*}\left(k_{1}, k_{2}, t\right) \\
& \times e^{-i k_{1} r_{1}-i k_{2} r_{2}} d k_{1} d k_{2} \sin \alpha d \alpha d \beta d \gamma,
\end{aligned}
$$

where

$$
k_{1} r_{1}+k_{2} r_{2}=\left(E_{1 q} k_{1}+E_{2 q} k_{2}\right) r_{q},
$$

and

$$
\begin{aligned}
\hat{U}_{l m}\left(k_{1}, k_{2}\right)= & \frac{1}{4 \pi^{2}} \int_{-\infty}^{\infty} \int_{-\infty}^{\infty} u_{l}\left(r_{1}, r_{2}, t\right) u_{m}\left(r_{1}, r_{2}, t\right) \\
& \times e^{-i k_{1} r_{1}-i k_{2} r_{2}} d r_{1} d r_{2}, \\
\hat{U}_{l}\left(k_{1}, k_{2}\right)= & \frac{1}{4 \pi^{2}} \int_{-\infty}^{\infty} \int_{-\infty}^{\infty} u_{l}\left(r_{1}, r_{2}, t\right) e^{-i k_{1} r_{1}-i k_{2} r_{2}} \\
& \times d r_{1} d r_{2},
\end{aligned}
$$

are the sectional Fourier transforms of $u_{l} u_{m}$ and $u_{l}$ respectively. We now transform to polar coordinates in vortex axes $k_{1}=k \cos \phi, k_{2}=k \sin \phi, r_{1}=r \cos \theta, r_{2}=r \sin \theta$ where $r$ is in the plane of the vortex and $k$ is modulus of the wave number in $k$-space. Note that we may put $k=|\boldsymbol{k}|=\mathrm{k}=|\mathbf{k}|$ owing to a prior integration over a shell of constant $k$ in vortex axes in the derivation of (B7)-(B9). When the choice $\mathbf{r}=(0,0, r)$ is made, and we use $E_{13}=-\sin \alpha \cos \gamma, E_{23}=\sin \alpha \sin \gamma$, on the right side of (B8) it is found that the exponentials in (B7) may be written as

$$
e^{-i k_{1} r_{1}-i k_{2} r_{2}}=e^{i k r \sin \alpha \cos (\gamma+\phi)} .
$$

The integrals over $\alpha, \beta$ and $\gamma$ in (B7) can now be done explicitly and it is found that

$$
\begin{gathered}
\int_{0}^{\pi} \int_{0}^{2 \pi} \int_{0}^{2 \pi} E_{13} e^{i k r \sin \alpha \cos (\gamma+\phi)} \sin \alpha d \alpha d \beta d \gamma \\
\quad=i 8 \pi^{2} \cos \phi\left(\frac{\cos (k r)}{k r}-\frac{\sin (k r)}{k^{2} r^{2}}\right),
\end{gathered}
$$

$$
\begin{gathered}
\int_{0}^{\pi} \int_{0}^{2 \pi} \int_{0}^{2 \pi} E_{23} e^{i k r \sin \alpha \cos (\gamma+\phi)} \sin \alpha d \alpha d \beta d \gamma \\
=i 8 \pi^{2} \sin \phi\left(\frac{\cos (k r)}{k r}-\frac{\sin (k r)}{k^{2} r^{2}}\right) .
\end{gathered}
$$

When (B11) and (B12) are used in (B7) and in the similar expressions for the other components of $B_{l 3, l}(\mathbf{r})$, we can obtain, after some algebra

$$
\begin{aligned}
B_{l 3, l}(0,0, r)= & i 4 \pi^{2} N \int_{t_{1}}^{t_{2}} e^{a t} d t \int_{0}^{\infty} \int_{0}^{2 \pi[} \cos \phi\left(\hat{U}_{11} \hat{U}_{1}^{*}\right. \\
& +\hat{U}_{12} \hat{U}_{1}^{*}+\sin \phi\left(\hat{U}_{12} \hat{U}_{2}^{*}+\hat{U}_{22} \hat{U}_{2}^{*}\right) \\
& \times\left(\frac{\cos (k r)}{k r}-\frac{\sin (k r)}{k^{2} r^{2}}\right) k d k d \phi .
\end{aligned}
$$

Comparison of (B13) with (B4) [using $\mathbf{r}=(0,0, r)]$ then gives

$$
\begin{aligned}
k^{2} F_{3}(k)= & i \frac{\pi N}{2} \int_{t_{1}}^{t_{2}} e^{a t} d t \int_{0}^{2 \pi}\left[\cos \phi\left(\hat{U}_{11} \hat{U}_{1}^{*}+\hat{U}_{12} \hat{U}_{1}^{*}\right)\right. \\
& \left.+\sin \phi\left(\hat{U}_{12} \hat{U}_{2}^{*}+\hat{U}_{22} \hat{U}_{2}^{*}\right)\right] k d \phi .
\end{aligned}
$$

When the right-side of (B14) is evaluated, $B_{3}(r)$ can be calculated from (B3). Equation (B14) can be expressed directly in terms of the Fourier expansion of the components of velocity induced by the vortex. The analysis leads to a complicated set of sums over integrals weighted by Bessel functions, $J_{n}(k r)$, of the Fourier components of $u_{\theta}$ and its square $u_{\theta}^{2}$. These can be estimated by stationary phase. Owing to the extreme complexity of the algebra we have not carried through this analysis for the stretched spiral vortex. The form of $F_{3}(k)$ which should be expected, on dynamical grounds, from such a calculation can nevertheless be inferred from the Kármán-Howarth equation in $k$-space. For decaying turbulence this is (Batchelor ${ }^{5}$, Monin and Yaglom ${ }^{15}$ )

$$
\frac{\partial E(k)}{\partial t}=-8 \pi k^{3} F_{3}(k)-2 \nu k^{2} E(k),
$$

where $E(k)$ is the shell-summed energy (velocity) spectrum. For stationary forced turbulence, the unsteady term can be replaced by the spectrum of forcing. For decaying turbulence at sufficiently high Reynolds numbers it is usually assumed that the unsteady term has large magnitude only within the energy containing range at small $k$. The term $-8 \pi k^{3} F_{3}(k)$ $=T(k)$ is the spectral transfer. At small wave numbers there is an approximate balance between $d E / d t$ and $T(k)\left(F_{3}\right.$ is positive) while in the dissipation range there is balance between $T(k)$ and dissipation ( $F_{3}$ is negative). Since the stretched-spiral vortex is a model of the fine scales, then for consistency with the Kármán-Howarth equation, a direct evaluation of the right-side of (B14) from a given velocity field would be expected to give

$$
F_{3}(k) \approx-\frac{1}{4 \pi} \nu k^{-1} E(k),
$$

in the universal equilibrium range of isotropic turbulence. 
At first sight it may appear that this is inconsistent with (14). But consider (12.141") of Monin and Yaglom ${ }^{15}$ [see also Batchelor, ${ }^{5}$ Equation (5.5.14)]

$$
K(r)=-8 \pi \int_{0}^{\infty} \frac{\sin k r}{k r} k^{3} F_{3}(k) d k,
$$

where

$$
K(r)=\frac{\partial B_{l j, l}(r)}{\partial r_{j}}=\frac{1}{6}\left(r \frac{\partial}{\partial r}+3\right)\left(\frac{1}{2} \frac{\partial}{\partial r}+\frac{2}{r}\right) B_{3}(r) .
$$

Adding and subtracting $k^{3} F_{3}(k)$ to the integrand of (B17) and using that

$$
\int_{0}^{\infty} k^{3} F_{3}(k) d k=0,
$$

(B17) may be written in the equivalent form

$$
K(r)=-8 \pi \int_{0}^{\infty}\left(\frac{\sin k r}{k r}-1\right) k^{3} F_{3}(k) d k .
$$

When $r$ lies in the inertial subrange, the main contribution to the integral in (B20) will come from large $k$. Thus, when (B16) is substituted into (B20) and the limit $\nu \rightarrow 0$ is taken, the contribution to the integral from the first term will be small giving $K(r) \approx-\epsilon$. When this is used in (B18) a particular integral gives $B_{3}(r)=-4 r \epsilon / 5$ in agreement with (14).

Alternatively, solving (B15) for $k^{3} F(k)$ and substituting into (B17) gives

$$
K(r)=\int_{0}^{\infty} \frac{\sin k r}{k r}\left(\frac{\partial E(k)}{\partial t}+2 \nu k^{2} E(k)\right) d k .
$$

Here, when $r$ is in the range of inertial scales and we let $\nu \rightarrow 0$, the second integral is small and the main contribution comes from the unsteady term which is finite only at small $k$. The approximation $\sin (k r) / k r \approx 1$ is justified, and using

$$
\epsilon=-\frac{d}{d t} \int_{0}^{\infty} E(k) d k
$$

we again find $K(r) \approx-\epsilon$ from which (14) follows. These arguments are model independent.

The apparent paradox, namely that the same result for $B_{3}(r)$ is obtained from the dominant terms of (B15) at both large and small wave numbers, is guaranteed by (B19). In the inertial subrange $F_{3}(k) \rightarrow 0$ when $\nu \rightarrow 0$ and so, unlike its physical space counterpart $B_{3}(r), F_{3}(k)$ does not conform to classical Kolmogorov inertial-range scaling except in a trivial sense in which the scaling constant is zero.

${ }^{1}$ D. I. Pullin and P. G. Saffman, "On the Lundgren-Townsend model of turbulent fine scales," Phys. Fluids A 5, 126 (1993).

${ }^{2}$ D. I. Pullin, J. D. Buntine, and P. G. Saffman, "On the spectrum of a stretched spiral vortex," Phys. Fluids 6, 3010 (1994).

${ }^{3}$ A. A. Townsend, "On the fine-scale structure of turbulence,"' Proc. R. Soc. Lond. Ser. A 208, 534 (1951).

${ }^{4}$ T. S. Lundgren, "Strained spiral vortex model for turbulent fine structure," Phys. Fluids 25, 2193 (1982).

${ }^{5} \mathrm{G}$. K Batchelor, The Theory of Homogeneous Turbulence (Cambridge University Press, Cambridge, 1953).

${ }^{6} \mathrm{~T}$. von Kármán and L. Howarth, "On the statistical theory of isotropic turbulence,"’ Proc. R. Soc. London, Ser. A 164, 192 (1938).

${ }^{7}$ L. D. Landau and E. M. Lifshitz, Fluid Mechanics (Addison-Wesley, New York, 1967)

${ }^{8}$ A. N. Kolmogorov, "Dissipation of energy in the locally isotropic turbulence,' Dokl. Akad. Nauk SSSR 32, (1941). Also Proc. R. Soc. London, Ser. A 434, 15 (1991).

${ }^{9}$ A. C. Robinson and P. G. Saffman, "Stability and structure of stretched vortices,' Stud. Appl. Math. 70, 163 (1984).

${ }^{10}$ H. K. Moffatt, S. Kida, and K. Ohkitani, "Stretched vortices-the sinews of turbulence-large Reynolds number asymptotics,', J. Fluid Mech. 259, 224 (1994).

${ }^{11} \mathrm{P}$. Tabeling (private communication).

${ }^{12}$ P. Tabeling, G. Zocchi, F. Belin, J. Maurer, and H. Williame, "Probability density functions, skewness and flatness in large Reynolds-number turbulence,' Phys. Rev. E 53, 1613 (1996).

${ }^{13}$ F. Anselmet, Y. Gagne, E. J. Hopfinger, and R. A. Antonia, "Higher-order velocity structure functions in turbulent shear flows," J. Fluid Mech. 140, 63 (1984).

${ }^{14}$ J. Jiménez, A. Wray, P. G. Saffman, and R. S. Rogallo, "The structure of intense vorticity in homogeneous isotropic turbulence," J. Fluid Mech. 255, 65 (1993)

${ }^{15}$ A. S. Monin and A. M. Yaglom, Statistical Fluid Mechanics, Vol. 2 (MIT Press, Cambridge, MA, 1975)

${ }^{16}$ D. I. Pullin and P. G. Saffman, "Reynolds stresses and one-dimensional spectra for a vortex model of homogeneous anisotropic turbulence,'” Phys. Fluids 6, 1787 (1994) 